\title{
Musculoskeletal lower-limb robot driven by multifilament muscles
}

\author{
Shunichi Kurumaya ${ }^{1 *}$, Koichi Suzumori ${ }^{1}$, Hiroyuki Nabae ${ }^{1}$ and Shuichi Wakimoto ${ }^{2}$
}

\begin{abstract}
This paper presents a redundant musculoskeletal robot using thin McKibben muscles that is based on human anatomy. The purpose of this robot is to achieve motions and characteristics that are very similar to a human body. We use a thin McKibben muscle, which is compliant and flexible, as the actuator of a musculoskeletal robot. Using a bundle of thin McKibben muscles, we develop a multifilament muscle that has characteristics similar to those of human muscles. In contrast, the actuators of conventional musculoskeletal robots are very heavy, not densely attached and have poor backdrivability. Because multifilament muscles are light and can be densely attached, we can attach them to the musculoskeletal robot as skeletal muscle and achieve a redundant system that is equivalent to a human drive mechanism. In this paper, we report a method for fabricating multifilament muscles that imitate various muscles, the development of a lower-limb muscle mechanism for the redundant musculoskeletal robot with thin McKibben muscles and experimental results showing that the proposed musculoskeletal robot achieves humanlike motions that have not yet been reported for other robots.
\end{abstract}

Keywords: Musculoskeletal robot, Pneumatic actuator, McKibben muscle, Biomimetics

\section{Background}

Currently, research on humanoid robots that imitate human drive mechanisms is vigorously carried out worldwide. Our research group believes that we can achieve humanlike behavior by imitating human mechanisms and structure perfectly using muscle placement, redundancy and tendon-driven systems. "Humanlike" mechanisms imply particular mechanisms that conventional robots do not have but a human has. These mechanisms make our robot more similar to a human than other robots. There are motions that are achieved by imitating human motions perfectly, for instance, knee rotation that appears only when the knee is bending or the complex bending motion of an ankle with its many degree of freedom. Many kinematic differences exist between presentday robots and human bodies. For example, (1) robotic knees typically consist of a revolute joint with a fixed rotational axis, while human knees consist of a rolling

\footnotetext{
*Correspondence: kurumaya.s.aa@m.titech.ac.jp

1 Department of Mechanical and Aerospace Engineering, Tokyo Institute of Technology, Meguro-ku, Ookayama 2-12-1, Tokyo 152-8550, Japan Full list of author information is available at the end of the article
}

joint with a shifting rotational axis; (2) robotic knees typically have one degree of freedom that supports bending, while human knees have two degrees of freedom at bending position that support bending and rotation; and (3) robotic ankles typically consist of a ball joint and a nondeformable foot, while human ankles consist of extrinsic muscles that not only support ankle motion but also deform the foot to a curved shape with inversion and eversion. These functional differences between presentday robots and human bodies also result in characteristic and physical appearance differences.

The final goal of this research was to further develop humanoid robots with human-like characteristics by imitating the human drive mechanism, including the number and arrangement of muscles in the human body. Human-like characteristics such as a deformable foot plays an important role in walking and knee rotation at a bending position contribute to operating the pedals of a car. Taking advantage of such human-like characteristics, our robot can be used to test hypotheses related to human motion as well as compare the performance of the robot to that of humans and work in the real world, e.g., as human interactive robots, amusement robots and 
medical training robots in the future. With this goal in mind, this paper describes efforts to verify the potential for human-like robotic mechanisms by building a similar drive mechanism using our unique thin muscles.

Musculoskeletal robots that have tendon-driven systems, mainly using motors, can better imitate the motions and characteristics of a human than those that have other drive mechanisms. Kenshiro [1, 2] is driven by motors and tendons. The body of Kenshiro is similar to that of a human because its muscles, bones and joint structures are based on human anatomy. For example, its knee joint is designed to imitate a human one; thus, Kenshiro has the functionality of a kneecap, cruciate ligament and screw-home mechanism in the knee joint using link mechanics, which allows for humanlike motion. ECCEROBOT (Embodied Cognition in a Compliantly Engineered Robot) $[3,4]$ is also driven by a tendon-driven system. It consists of a skeleton made from a polymorph that has a bone-like appearance and elastic actuators that include motors and elastic tendons to realize motions that are similar to a human. ECCEROBOT is used to test hypotheses about human motion as well as compare its performance with that of humans. However, the actuators of these conventional musculoskeletal robots with motor-driven tendon mechanisms are very heavy, not densely attached to the muscles and have poor backdrivability with respect to the motors, gear wheels and belts. Therefore, these robots do not have a redundancy that is as good as that of a human.

A McKibben artificial muscle is an actuator that has an elasticity and compliance that is similar to human muscle and can also be used in a tendon-driven mechanism. According to [5], the Shadow Biped Walker, which is a pioneering redundant robot with pneumatic muscles, has been developed by Shadow Robot Co. in 1988. The muscle arrangement in the robot is similar to that of human muscles; however, the number of muscles on its one leg is only 14 [6]. Koh Hosoda et al. have developed musculoskeletal infant robots with pneumatic artificial muscles [7]. These robots have a humanlike musculoskeletal structure and McKibben pneumatic muscles that imitate human muscles. Compared with motors, these actuators have some advantages regarding their mechanical softness and compliance. As a result, such robots are a good platform for investigating motion development. In addition, the same research group also developed a biped robot powered by antagonistic pneumatic actuators [8]. The design concept of this robot is basically the same as that of the robots in [7]. This robot suggests that joint compliance contributes to the realization of various types of locomotion. The humanoid muscle robot torso called "Zwei-Arm-Roboter" has been developed based on an idea that is similar to ours [9]. Developers insist that biologically inspired robots embody no rigid movement, which are made possible by special joints or actuators. However, these pneumatic actuator robots do not perfectly imitate the redundancy of the human drive system with respect to the number of muscles for the same reason that Kenshiro and ECCEROBOT do not.

We have developed a thin McKibben muscle [10] with a flexible shape that is the thinnest McKibben muscle ever reported. This actuator is also lighter and more compact than other actuators such as conventional pneumatic muscles, motors and cylinders. We believe that our unique thin muscle is currently the only actuator capable of realizing this purpose: it is thin, deformable and light enough to be used inside a body with limited space; it generates a comparable contracting force and ratio as human muscles; and it can be easily bundled to form various shaped multifilament muscles, e.g., a muscle that has two ends on one side, such as a biceps muscle, or a flat muscle such as the pectoralis major and deltoid muscles. Kenshiro [1, 2] has a flat shaped (or planar) muscle [11]; however, this planar muscle requires several pulleys and associated supports. Comparatively, the shape and acting points of a multifilament muscle can be flexibly changed and adjusted during implementation in a robot body. As a result, these muscles can be densely attached in the robot. By replacing conventional actuators with thin McKibben muscles on a musculoskeletal robot, we can use them more densely and build a musculoskeletal robot with a perfectly humanlike redundant system, resulting in characteristics that are more similar to a human than other systems.

In this paper, we report a new method for fabricating multifilament muscles that imitate various muscles, the development of a lower-limb muscle mechanism with the same number of muscles as a human for a musculoskeletal lower-limb robot driven by these multifilament muscles (Fig. 1), and results that compare the prototype's motions with that of a human. The experimental results showed that our robot successfully realized human-like knee and ankle motions with foot deformation that have not been previously realized using conventional actuators and robots.

\section{Thin McKibben muscle}

We are able to mass-manufacture the thin McKibben muscles, as shown in Fig. 2. The outer diameter of the thin McKibben muscle is $1.8 \mathrm{~mm}$, which is currently the thinnest of all McKibben artificial muscles. This actuator is made of a silicone tube with a hardness of 40 shore $\mathrm{A}$, $1.3 \mathrm{~mm}$ outer diameter and $0.9 \mathrm{~mm}$ inner diameter. The outer-sleeve yarn is made up of $0.12 \mathrm{~mm}$ Tetoron monofilament. The design specifications of the thin McKibben muscle used in this study include a braiding angle 


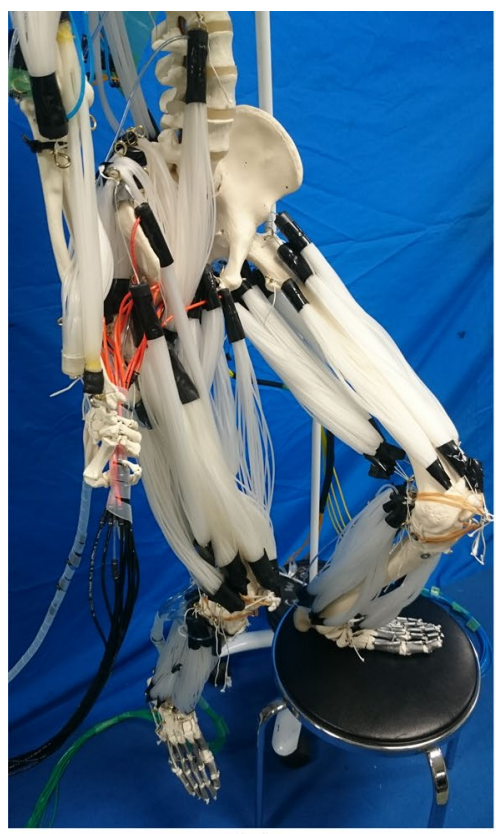

(a)
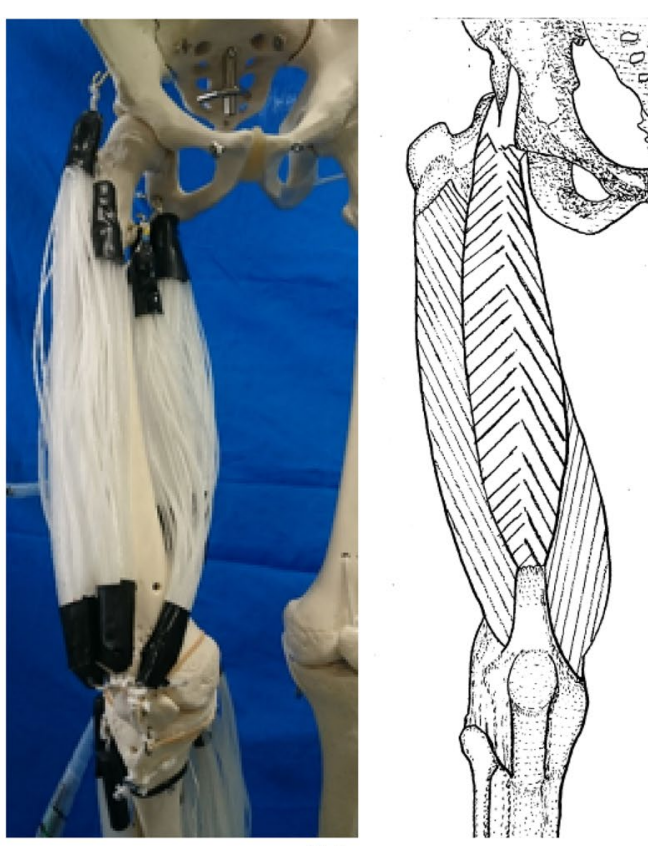

(b)

Fig. 1 a Redundant musculoskeletal robot with thin McKibben muscles. b Robotic and human quadriceps muscles. The right panel of $\mathbf{b}$ is drawn by the authors based on several anatomy texts such as [12]

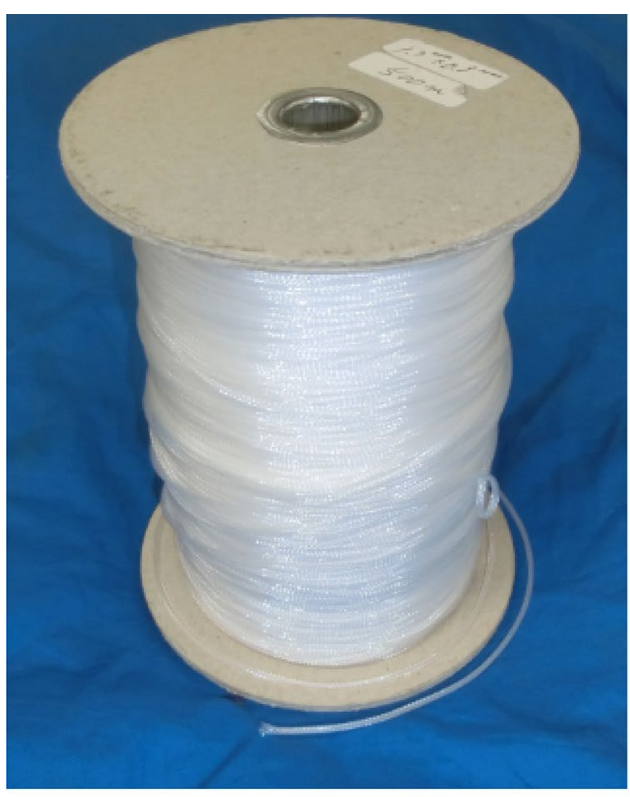

(a) Mass-manufactured thin McKibben muscle

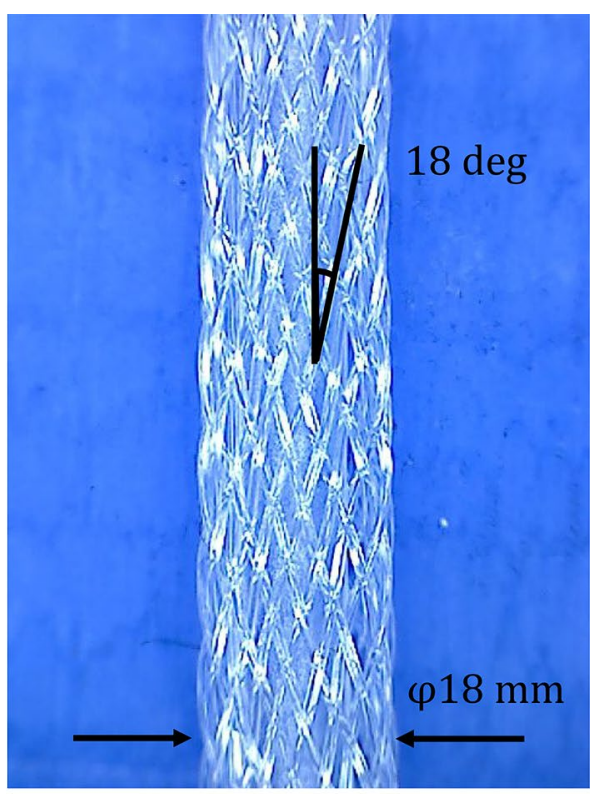

(b) Thin McKibben muscle detail

Fig. 2 a Roll of thin McKibben muscle (500 $\mathrm{m}$ in length). b Magnified view of thin McKibben muscle

of $18^{\circ}$ with 24 outer fibers. The characteristics of the thin McKibben muscles are shown in Fig. 3. It has hysteresis because of the rubber properties. The maximum contraction force is $11 \mathrm{~N}$ and the maximum contraction ratio is $24 \%$ with an air pressure of $0.35 \mathrm{MPa}$. While the first drive of the muscle displays a relatively 


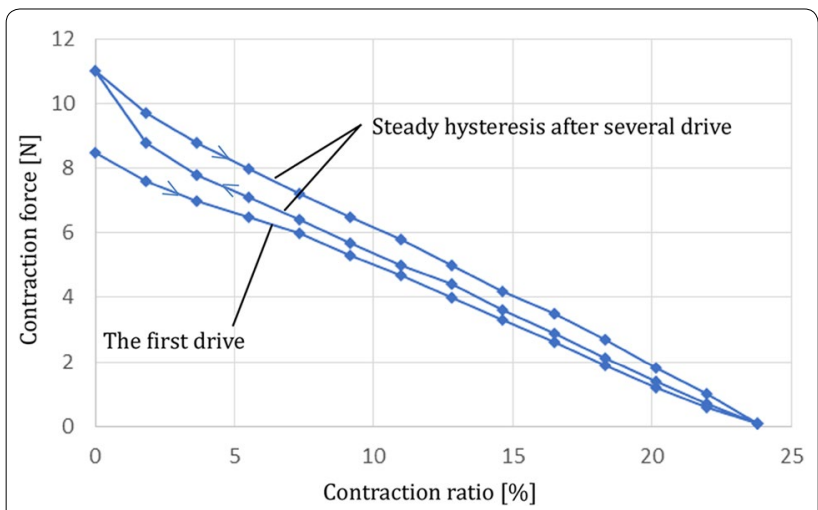

Fig. 3 Static characteristics of thin McKibben muscle with an air pressure of $0.35 \mathrm{MPa}$, where the maximum contraction force is $11 \mathrm{~N}$ and the maximum contraction ratio is $24 \%$

low contraction force because of the virgin hardness of the rubber and the friction between the sleeve yarn and the silicone tube, after several drive cycles, it exhibits a steady hysteresis property, as shown in Fig. 3.

\section{Design of the multifilament muscles} Structure of the multifilament muscle

The typical structure of the multifilament muscle is illustrated in Fig. 4. It has a tendon at each end and can be connected to a skeleton. One end has a tendon that is made of Dyneema (high-density polyethylene) fibers and an air-supply port that is typically made of heat-shrink plastic. The other end has a tendon only. This quick and easy fabrication method achieves a small and soft airsupply port with high sealing properties.

\section{Characteristics of the multifilament muscle}

The multifilament muscle basically works as a contracting linear actuator, as shown in Fig. 5. This muscle consists of 60 thin McKibben muscles, $310 \mathrm{~mm}$ in length and its contraction ratio is $20 \%$ at an air pressure of $0.25 \mathrm{MPa}$. It has been reported by Doi et al. [13] that the characteristics of multifilament muscles resemble those of thin McKibben muscles and its contracting force is nearly proportional to the number of muscles, while the contraction ratio is similar to that of a single thin McKibben muscle. Thus, we can easily design a muscle that has the desired property. Generally, the contracting force of the McKibben muscle is proportional to its cross-sectional area. Thus, the contracting force of a multifilament muscle is lower than that of a conventional muscle with the same diameter because of the dead space between muscles. This actuator is robust: if some of the thin McKibben muscles that compose the multifilament muscle are broken, the muscle continues to work with a little air leakage. We can repair and use it again by sealing that part. In this research, actuators have been designed and fabricated to be used as human muscles, which have a maximum contraction stress of $0.30 \mathrm{MPa}$ and a maximum contraction ratio of 25-30\%. In comparison with human muscle, the contraction ratio of multifilament muscle is almost same as that of human muscles and the contraction force is approximately 10 times higher or more. On the other hand, responsiveness is different between multifilament muscles and human muscles. To make the responsiveness of multifilament muscles as quick as that of human muscles, the opening area of valves and the length of air supply tube need to be improved.

\section{Various shapes of multifilament muscles}

Human muscles have various shapes, e.g., a muscle that has two ends on one side, such as a biceps muscle, or a flat muscle such as the pectoralis major and deltoid muscles, as shown in Fig. 6. Thus, it is difficult for conventional actuators alone to imitate the shapes and the functions of such muscles; however, multifilament muscles can imitate not only the shapes of human muscles, but also their functionality. The advantages of these muscles are that several points of application for a force are easily changed and output force increases in proportion to the number of muscles. Furthermore, a unique method of attaching

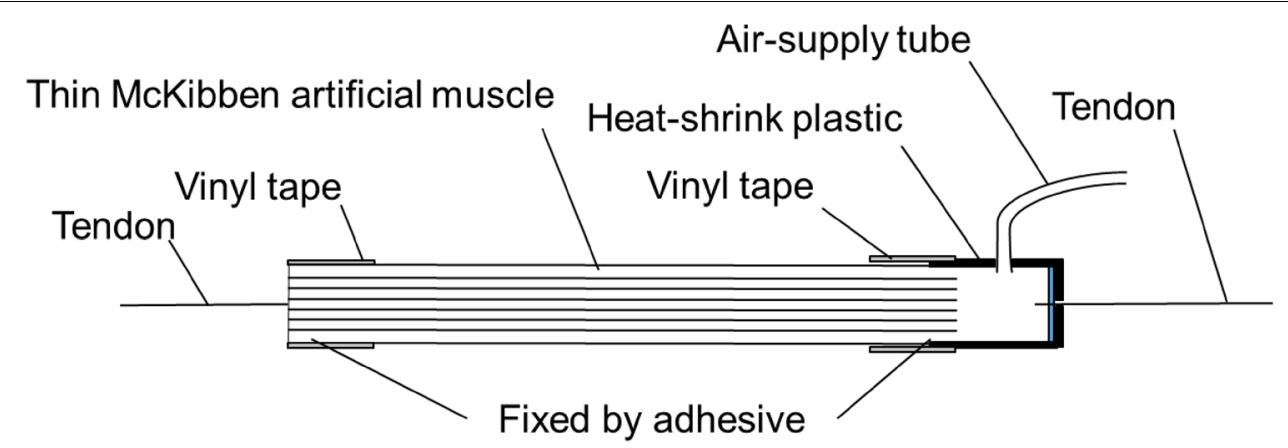

Fig. 4 Structure of typical multifilament muscle 


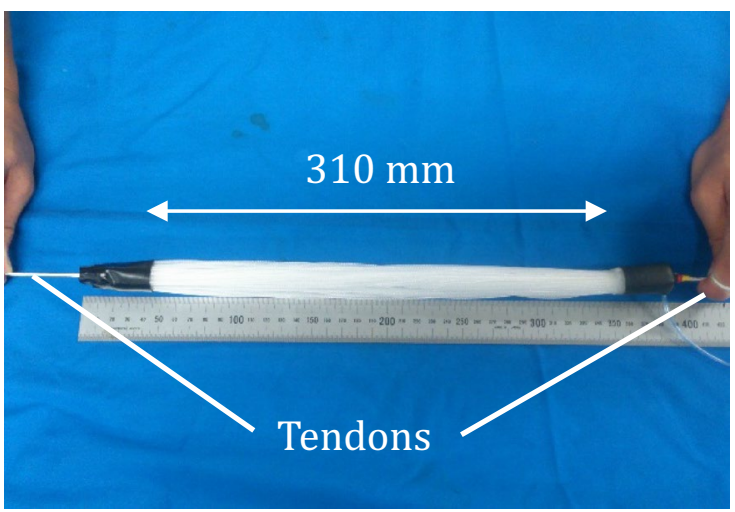

(a) Air pressure $0 \mathrm{MPa}$

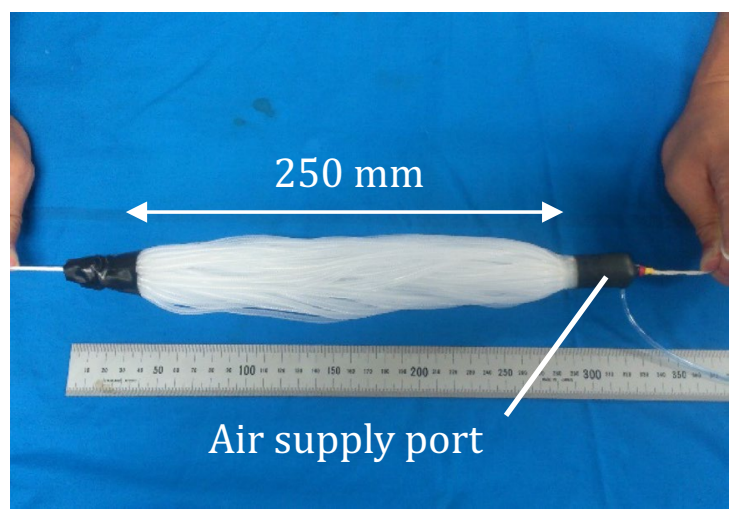

(b) Air pressure 0.25 MPa

Fig. 5 a Example of the developed multifilament muscle consisting of 60 thin Mckibben muscles working as a linear actuator. This is the basic shape that imitates normal muscle. b The contraction ratio is $20 \%$ when the air pressure is $0.25 \mathrm{MPa}$

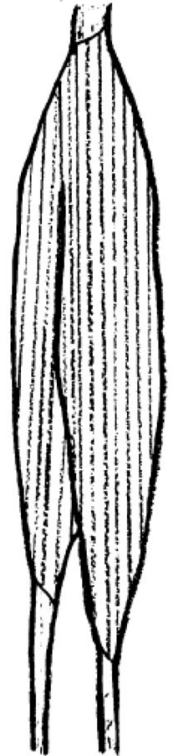

(a)

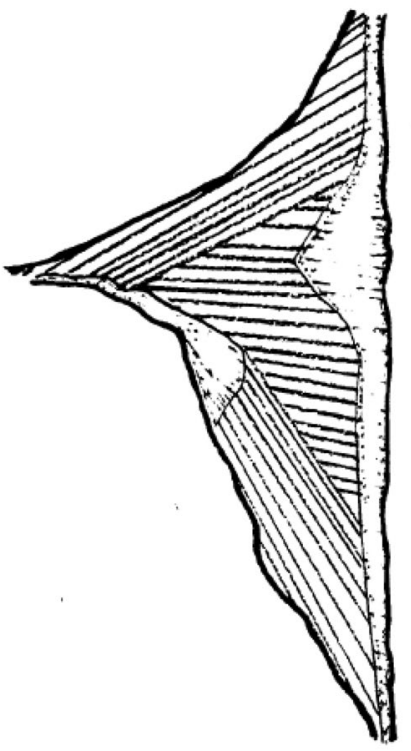

(b)
Fig. 6 Various human muscles. a Biceps muscle and $\mathbf{b}$ flat muscle such as a deltoid muscle (drawn by the authors based on several anatomy texts such as [7])

the actuator to the robot is achieved. Compared with the shaped muscle in [8], both ends of the muscle are small and each thin McKibben muscle is decentralized because of this bundling structure. To attach these actuators to a musculoskeletal robot and demonstrate the possibility of building various shapes, we fabricated them as a biceps muscle, as shown in Fig. 7. This muscle consists of 60 thin McKibben muscles, its length is $210 \mathrm{~mm}$ and its contraction ratio is $20 \%$ at an air pressure of $0.25 \mathrm{MPa}$ with a load of $50 \mathrm{~N}$ attached at the bottom. Another example we have fabricated is the flat muscle shown in Fig. 8. This muscle consists of 16 thin McKibben muscles, its length is $280 \mathrm{~mm}$ and its contraction ratio is $19 \%$ at an air pressure $0.25 \mathrm{MPa}$ with a load of $25 \mathrm{~N}$ attached at the bottom.

\section{Design of a lower limb musculoskeletal robot}

In this research, we used a skeletal specimen that imitates a boy $1.6 \mathrm{~m}$ in height, usually used as an anatomical model of the human skeleton in a hospital or science room, as the body of the musculoskeletal robot. We obtained several advantages by using it as the body of the musculoskeletal robot. For example, most conventional musculoskeletal robots have a shaft in the knee joints with a fixed rotational axis; however, the human knee has no fixed rotational shaft and rolls with a shifting rotational center. Kenshiro realized this motion by using a linkage mechanism, which is a very different structure than that of the human knee. The skeletal specimen we used has the same structure in the joint as a human, where the bones roll in contact with each other. Additionally, the skeletal specimen has ligaments made of elastic cord. With such features, the skeletal specimen can move more like a human than conventional robot linkage mechanisms.

\section{Design of knee motions}

Table 1 summarizes the design specifications of the multifilament muscles for the knee. The number of thin McKibben muscles is determined so that multifilament muscles are as almost the same size as human ones by 


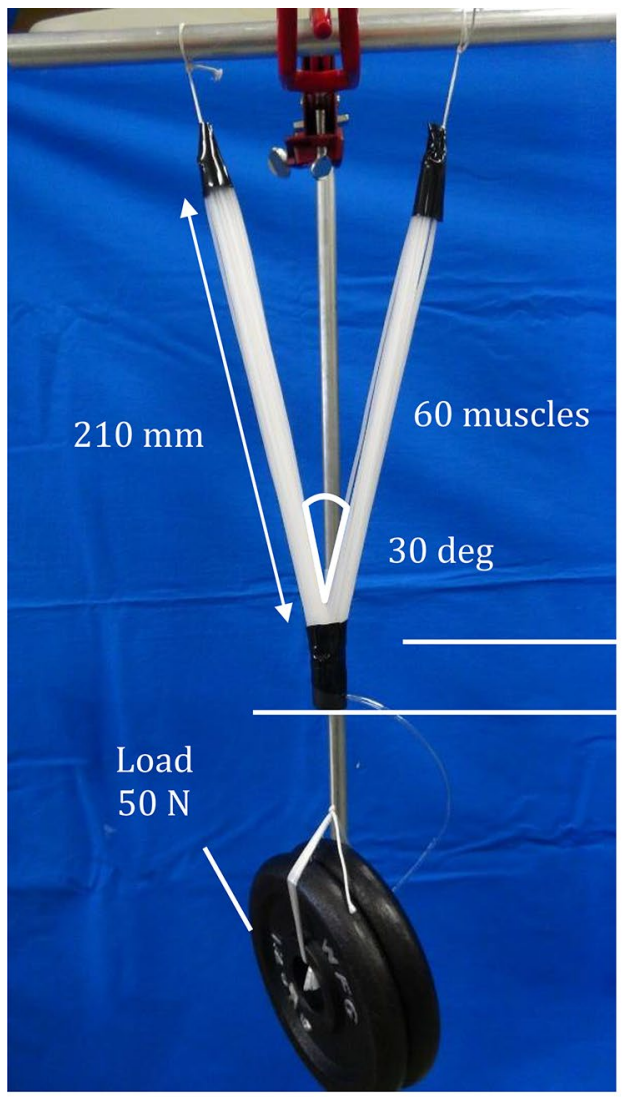

(a) Air pressure $0 \mathrm{MPa}$

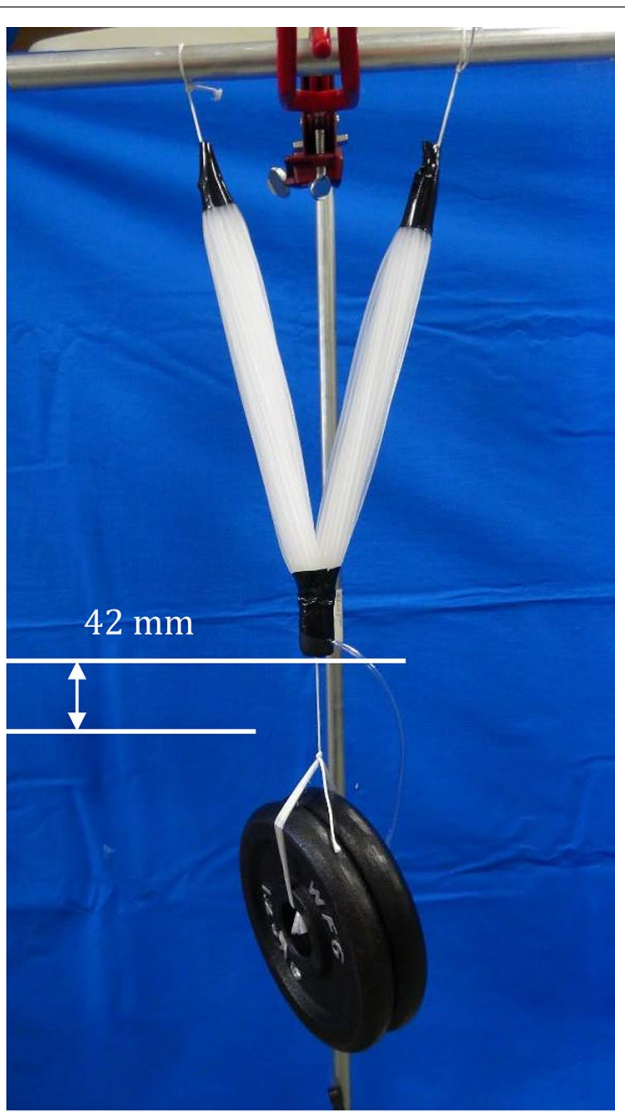

(b) Air pressure $0.25 \mathrm{MPa}$

Fig. 7 a Example of multifilament muscle consisting of 60 thin McKibben muscles that imitates a biceps muscle. b The contraction ratio is $20 \%$ at an air pressure of $0.25 \mathrm{MPa}$ with a $50 \mathrm{~N}$ load attached at the bottom

referring pictures and figures of anatomy manuals [14, 15]. We have confirmed that there is no significant difference between our muscles and human muscles under the supervision of a surgeon. In this paper, we do not have a quantitative discussion. Their lengths are determined by accounting for the height of the skeletal specimen used.

There are four types of knee motions: extension, flexion, internal rotation and external rotation. The muscles needed for each motion based on human anatomy are shown in Fig. 9. The extension of the knee uses four muscles: the rectus femoris, vastus lateralis, vastus intermedius and vastus medialis muscles. The rectus femoris muscle, which is a biarticular muscle, also acts upon the flexion of the hip joint. The flexion of the knee uses three muscles: the biceps femoris, semitendinosus and Semimembranosus muscles. Internal rotation uses the semitendinosus and Semimembranosus muscles. External rotation uses the biceps femoris muscle. Internal rotation and external rotation are carried out using only the flexion. This is why both muscles acting upon these motions also act upon the flexion.

\section{Design of ankle motions}

Table 2 also summarizes the design specifications of the multifilament muscles for the ankle. The number of thin McKibben muscles and their lengths are determined in the same manner as the knee.

There are four types of ankle motions: dorsal flexion, plantar flexion, inversion and eversion. In reality, there are six types of ankle motions: dorsal flexion, plantar flexion, pronation, supination, adduction and abduction. However, the latter four motions are included in inversion or eversion because of the limitations caused by muscle interactions. Inversion is an action that includes plantar flexion, supination and adduction. The muscles used for each motion based on human anatomy are shown in Fig. 10. Eversion is an action that includes dorsal flexion, pronation and abduction. Dorsal flexion uses four muscles: the tibialis anterior, fibularis tertius, extensor digitorum longus and extensor hallucis longus. Plantar flexion uses seven muscles: the gastrocnemius, soleus, plantaris, tibialis posterior, fibularis longus, fibularis brevis and flexor hallucis longus. The gastrocnemius also 


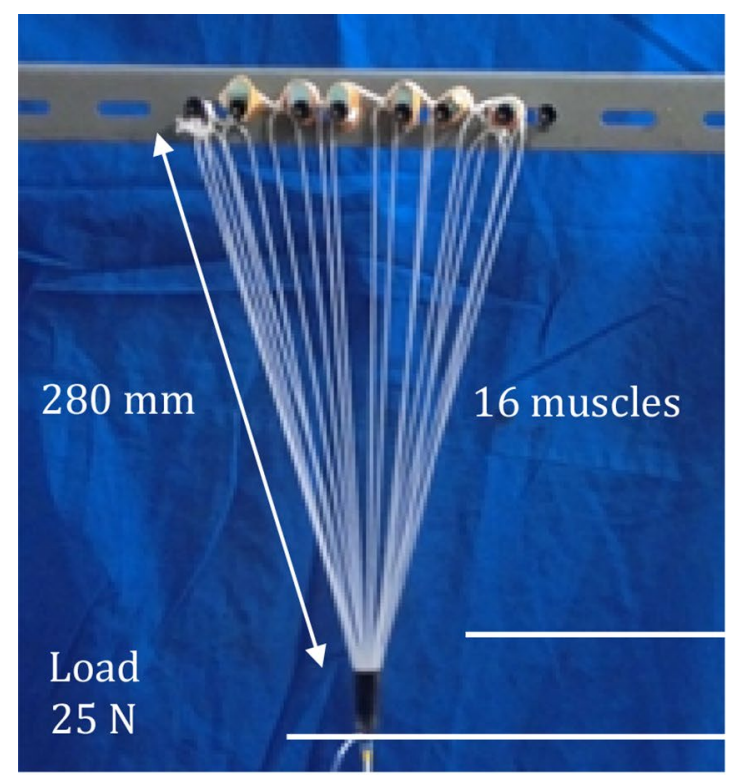

(a) Air pressure $0 \mathrm{MPa}$

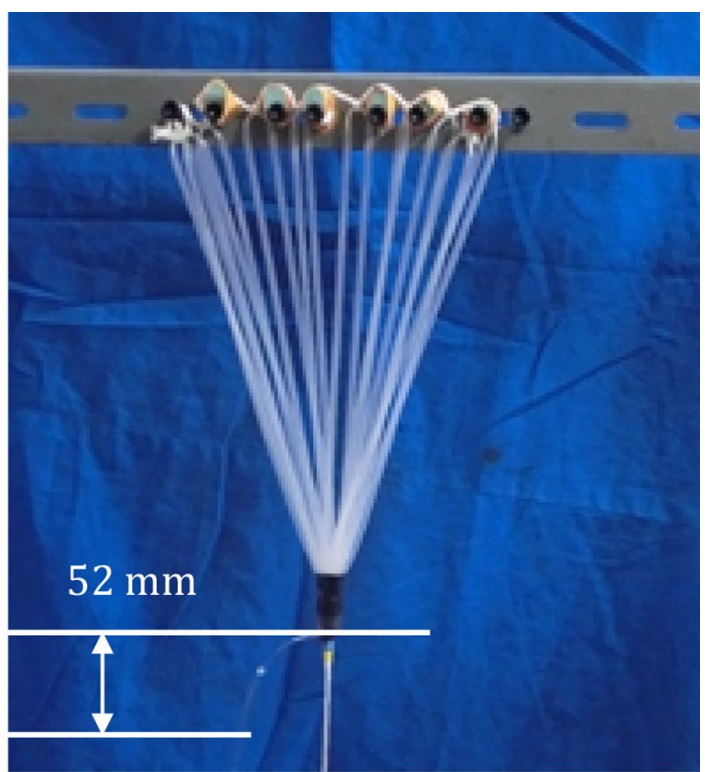

(b) Air pressure $0.25 \mathrm{MPa}$

Fig. 8 a Example of multifilament muscle consisting of 16 thin McKibben muscles that imitates a flat muscle. b The contraction ratio is $19 \%$ at an air pressure of $0.25 \mathrm{MPa}$ with a $25 \mathrm{~N}$ load attached at the bottom

Table 1 Design specifications of the multifilament thin McKibben muscles for the knee

\begin{tabular}{lll}
\hline Muscle & $\begin{array}{l}\text { Number of thin McK- } \\
\text { ibben muscles }\end{array}$ & $\begin{array}{l}\text { Length of con- } \\
\text { striction (mm) }\end{array}$ \\
\hline Rectus femoris & 65 & 325 \\
Vastus lateralis & 63 & 270 \\
Vastus intermedius & 48 & 270 \\
Vastus medialis & 60 & 300 \\
Biceps femoris (long one) & 60 & 250 \\
Biceps femoris (short one) & 72 & 280 \\
Semitendinosus & 60 & 350 \\
Semimembranosus & 60 & 250 \\
\hline
\end{tabular}

acts upon the flexion of the knee joint, which is a biarticular muscle. Inversion uses four muscles: the tibialis anterior, tibialis posterior, flexor digitorum longus and flexor hallucis longus. Eversion uses four muscles: fibularis tertius, fibularis longus, fibularis brevis and extensor digitorum longus.

\section{Development of a lower limb musculoskeletal robot}

Our musculoskeletal robot in Fig. 11 has eight multifilament muscles that affect knee motions on the thigh and 12 muscles that affect ankle motions on the lower leg, where the shape and size of the multifilament muscles are almost equivalent to those of a human. By fabricating various types of multifilament muscles, we have achieved the same structure as a human by combining the thin artificial muscles with a skeletal specimen. It is a redundant system, in which 20 multifilament muscles work independently. We attached the multifilament muscles to the musculoskeletal robot considering the fixed ends and the directions of the tendons. The sheath of a tendon, made of a soft tube, makes the tendon move smoothly.

The control system for the thin McKibben muscles is shown in Fig. 12. The multifilament muscles on the robot are provided with air pressure through a regulator and solenoid valves. The air pressure is controlled by a single regulator and the muscles on our musculoskeletal robot are operated by ON/OFF control solenoid valves.

\section{Motion evaluation results}

We evaluated our redundant musculoskeletal robot over a range of motions. Each motion is generated by activating the corresponding muscles based on human anatomy. Corresponding muscles are operated by ON/OFF control solenoid valves. We measured the range of motion for each robot joint and compared it 


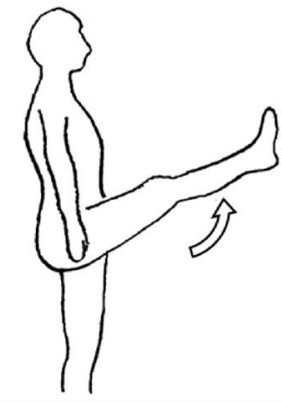

Extension

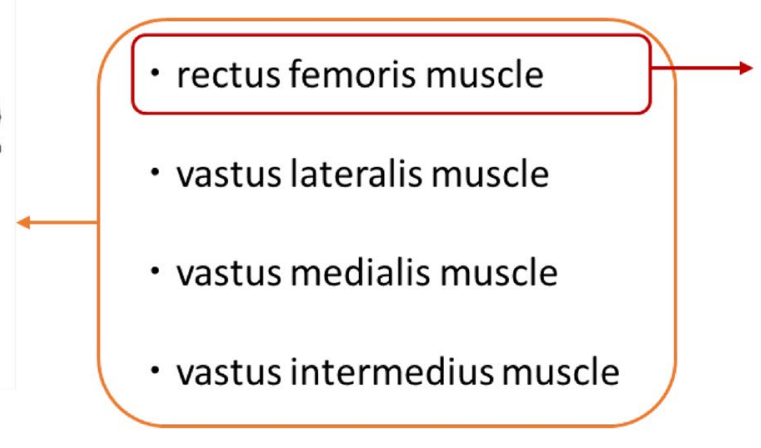

\section{Flexion of a hip joint}

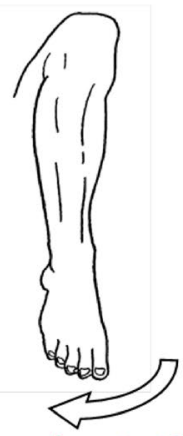

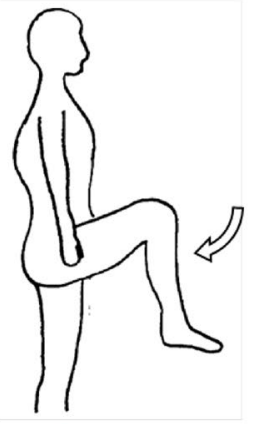

Flexion

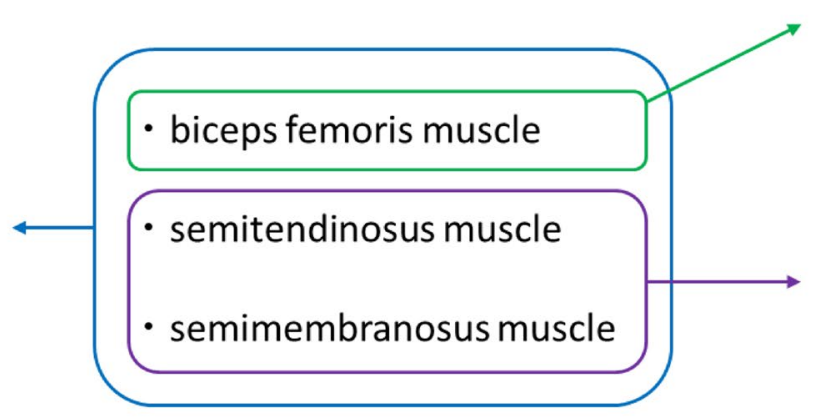

\section{External rotation}

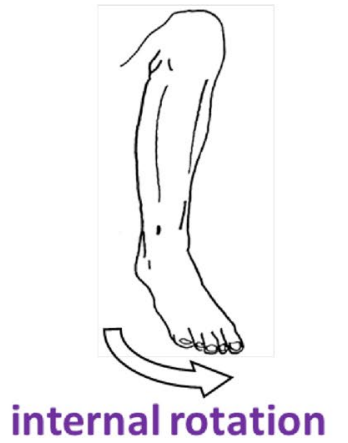

Fig. 9 Classification of the muscles used for each motion of the knee based on human anatomy

Table 2 Design specifications of the multifilament thin McKibben muscles for the ankle

\begin{tabular}{lll}
\hline Muscle & $\begin{array}{l}\text { Number of thin } \\
\text { McKibben muscles }\end{array}$ & $\begin{array}{l}\text { Length of } \\
\text { constriction (mm) }\end{array}$ \\
\hline Gastrocnemius & 30 each & 300 \\
Soleus & 40 & 300 \\
Plantaris & 10 & 300 \\
Tibialis anterior & 40 & 300 \\
Tibialis posterior & 26 & 300 \\
Fibularis tertius & 10 & 100 \\
Fibularis longus & 30 & 300 \\
Fibularis brevis & 40 & 160 \\
Flexor digitorum longus & 30 & 300 \\
Extensor digitorum longus & 30 & 300 \\
Flexor hallucis longus & 20 & 250 \\
Extensor hallucis longus & 20 & 250 \\
\hline
\end{tabular}

that of the corresponding human joint. Each angle of joint is obtained by measuring the intersection angles between bones in the pictures shown in Figs. 13, 14, $15,16,17$.

\section{Knee motion evaluation}

The extension and flexion of our musculoskeletal robot were confirmed, as shown in Fig. 13, to be similar to those of a human. The internal rotation and external rotations were also confirmed, as shown in Fig. 14. By comparing the range of motion of our musculoskeletal robot with that of a human (Tables 3 and 4), we find that the internal and external rotations of our robot are equal to those of a human. At the same time, the extension and flexion of our robot are about degree less than those of a human. 


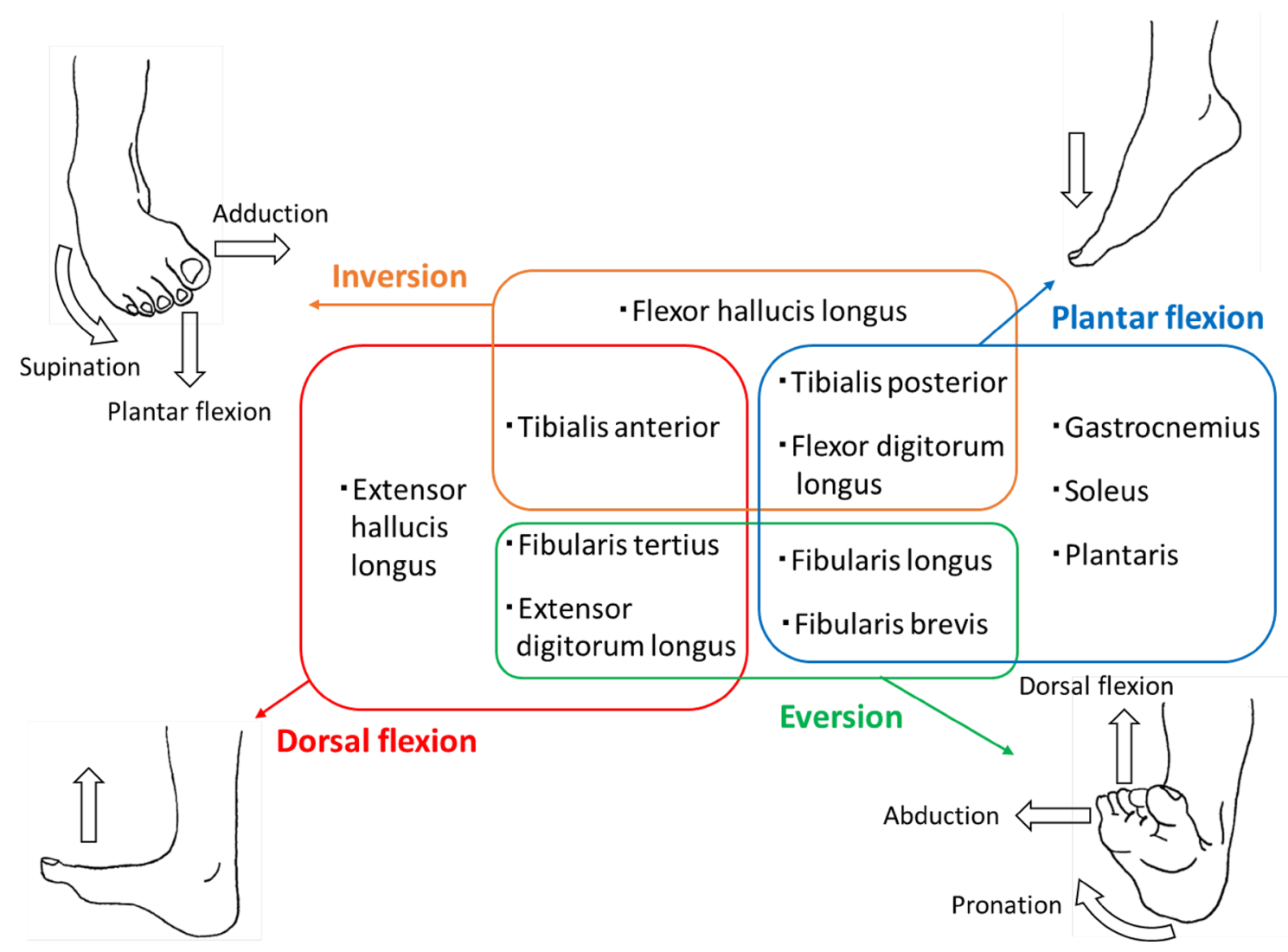

Fig. 10 Classification of the muscles used for each motion of the ankle based on human anatomy

\section{Ankle motion evaluation}

The dorsal and plantar flexions of our musculoskeletal robot were confirmed, as shown in Fig. 15, to be similar to those of a human. Furthermore, pronation and supination were confirmed, as shown in Fig. 16. Adduction and abduction during inversion or eversion were confirmed, as shown in Fig. 17, and are no less than those of a human. Inversion and eversion were also confirmed, as shown in Fig. 18. The comparison of the range of motions of our musculoskeletal robot with those of a human (Table 5) shows that except for dorsal flexion, the ranges are no less than those of a human. The plantar flexion of our robot is not more than that of a human; however, nearly the same motion as a human is achieved.
Another distinct point of the prototype is its foot deformation. As shown in Figs. 15 and 16, the prototype foot deforms in a curved shape, very similar to a human foot. This comes from the bone structure, which, like that of a human, consists of many bones, like a cubic puzzle.

\section{Conclusions}

We established a method for fabricating multifilament muscles using the thin McKibben muscle that we previously developed. We have also successfully imitated various-shaped muscles that are found in the human body. Using the multifilament muscles, this report shows that we can create redundant and compact tendon-driven 


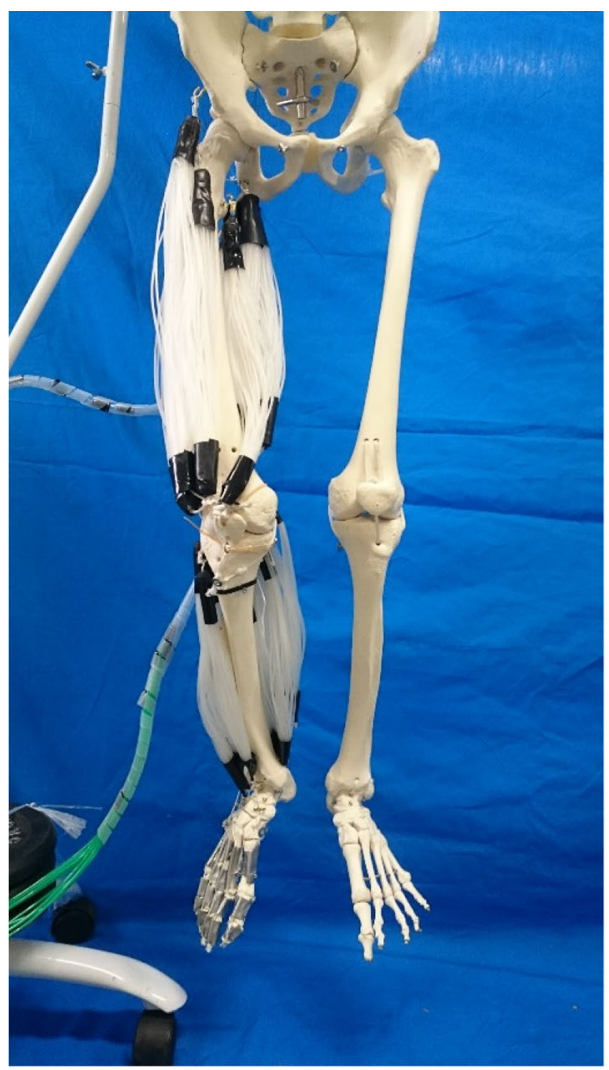

(a) Front view

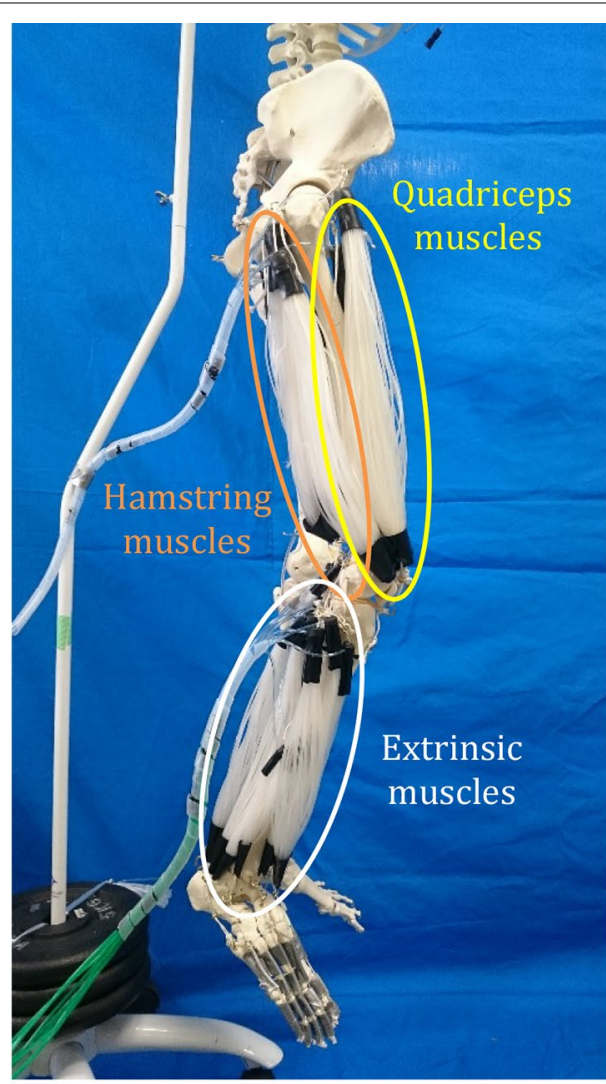

(b) Side view

Fig. 11 a Front and $\mathbf{b}$ side view of redundant musculoskeletal robot with thin McKibben muscles. There are eight multifilament muscles on the thigh and 12 muscles on the lower leg, which is equivalent to a human

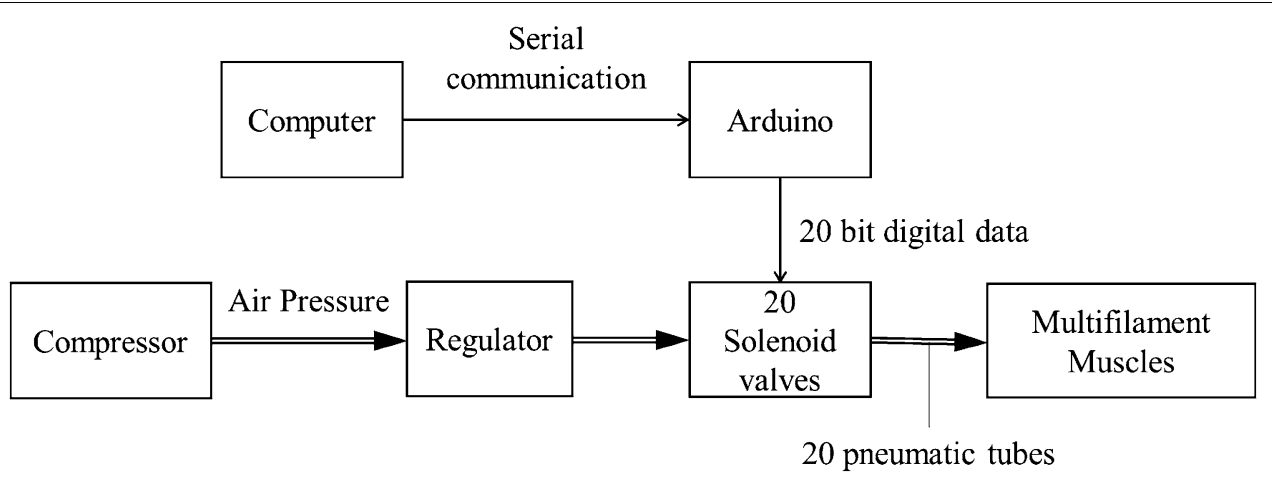

Fig. 12 Robot control system for the thin McKibben muscles based on an Arduino board. The multifilament muscles on the robot are provided with air pressure through a regulator and solenoid valves 


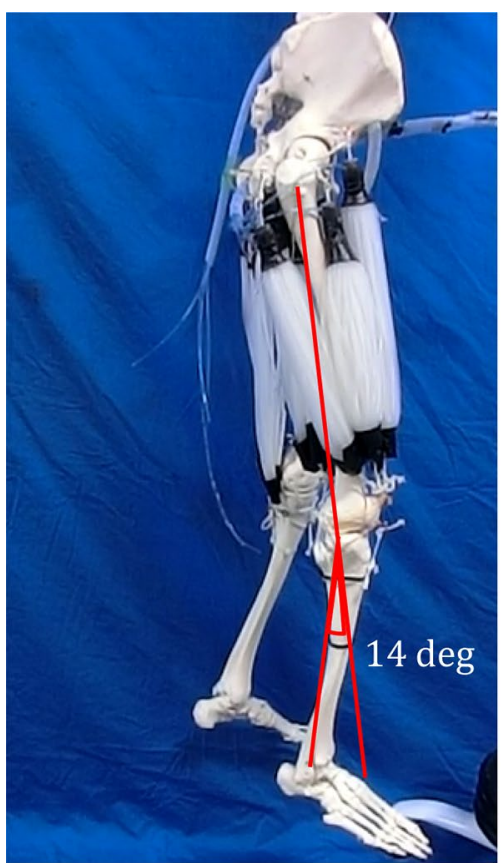

(a)

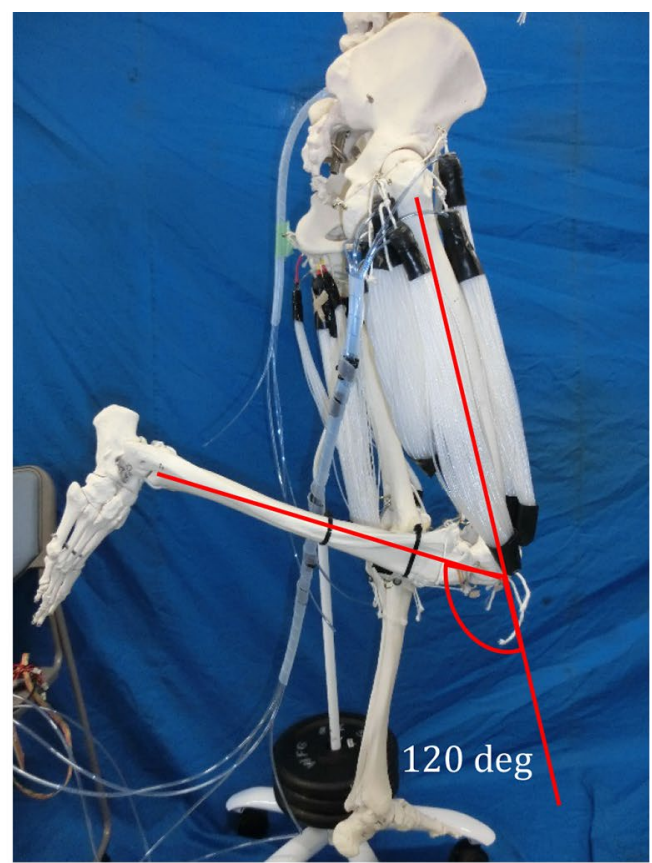

(b)

Fig. 13 a Extension and $\mathbf{b}$ flexion of the knee driven by multifilament muscles

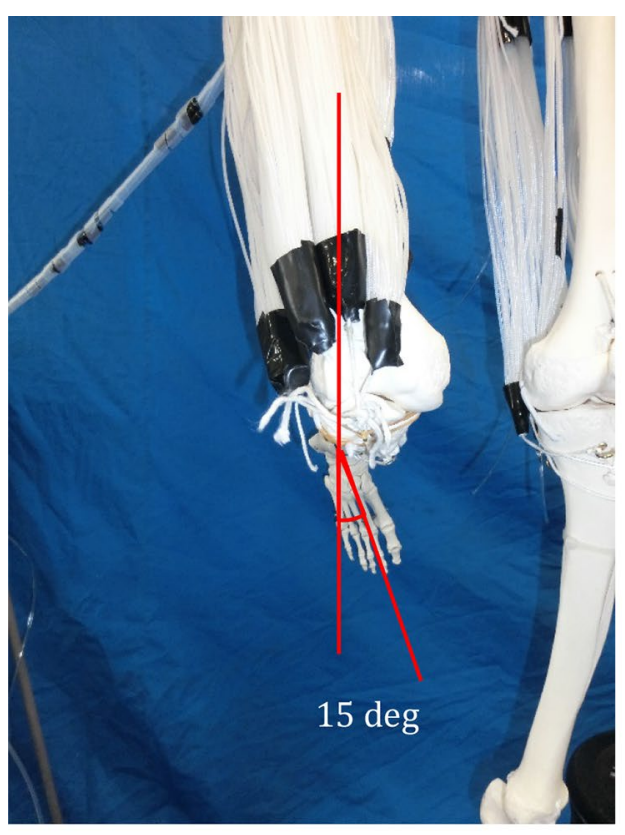

(a)

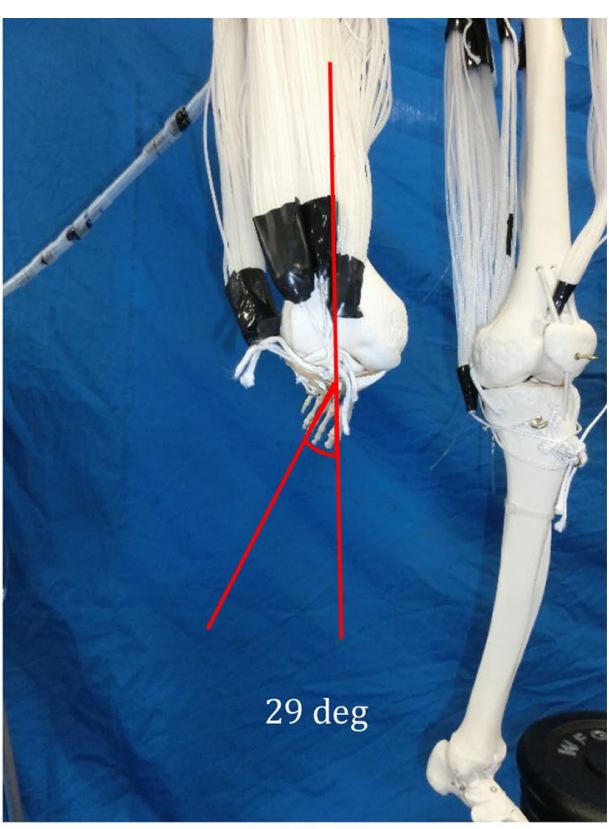

(b)

Fig. 14 a Internal rotation and $\mathbf{b}$ external rotation of the knee driven by multifilament muscles 


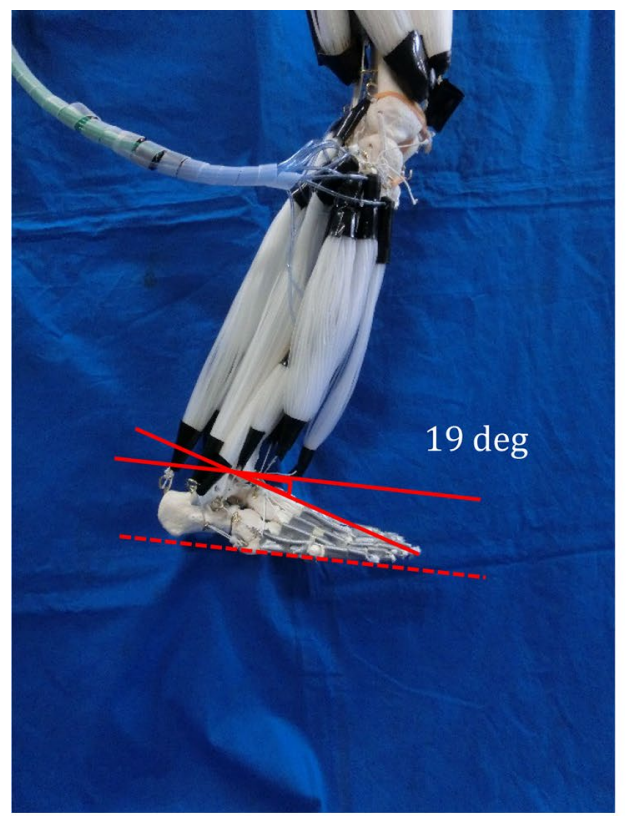

(a)

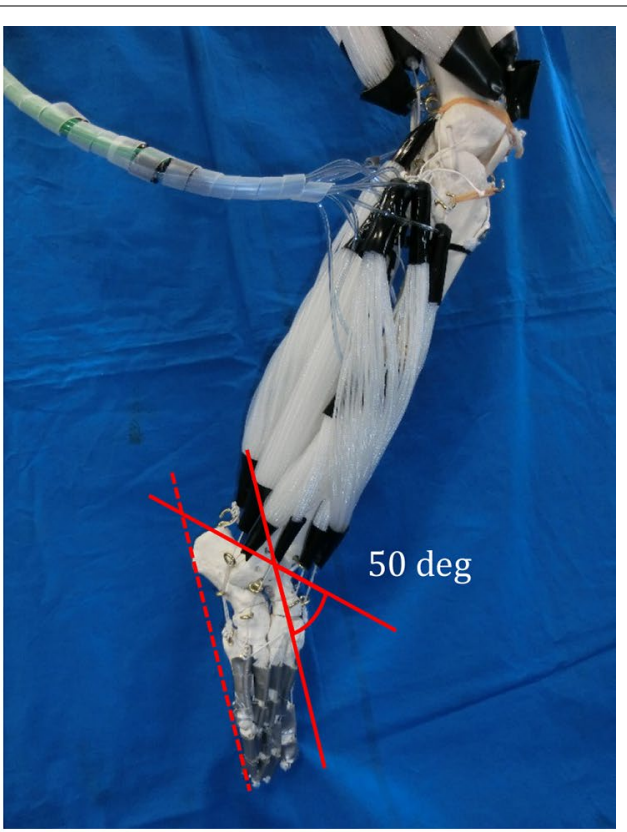

(b)

Fig. 15 a Dorsal flexion and $\mathbf{b}$ plantar flexion of an ankle driven by multifilament muscles

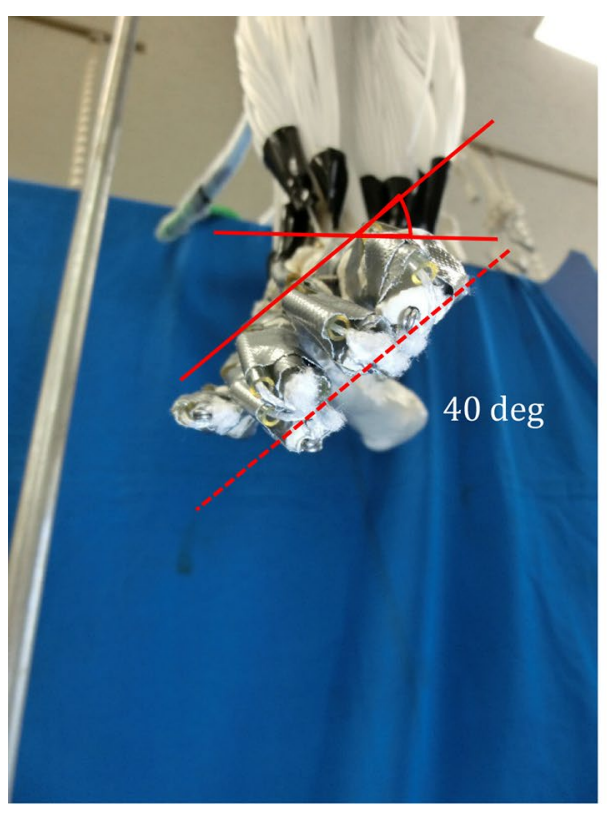

(a)

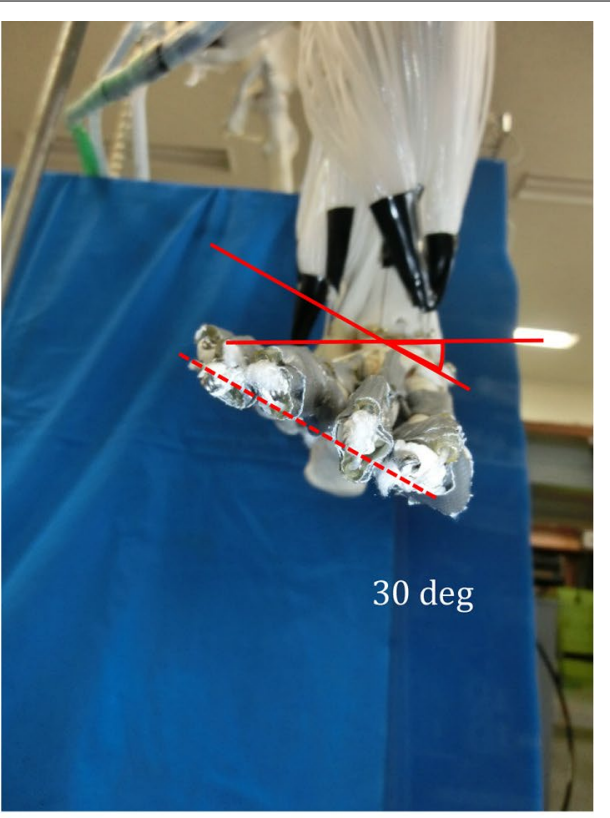

(b)

Fig. 16 a Pronation and $\mathbf{b}$ supination of an ankle driven by multifilament muscles during inversion or eversion 


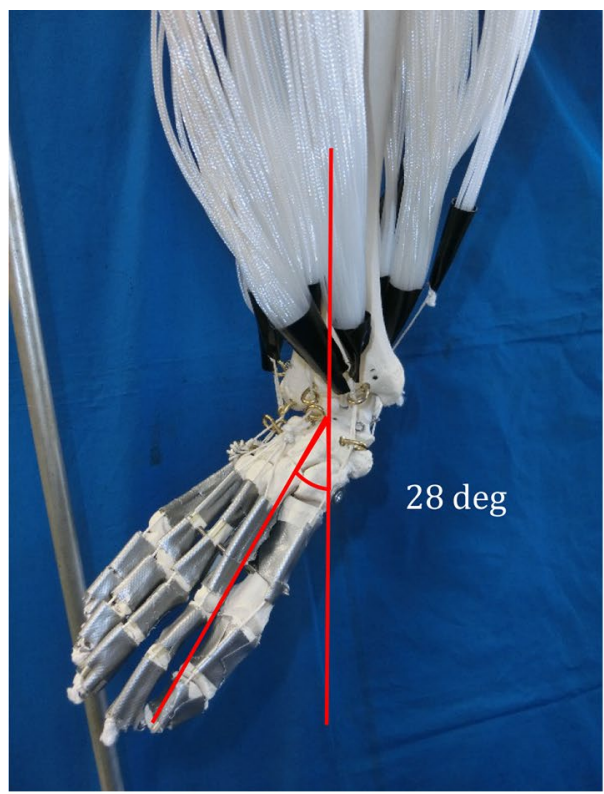

(a)

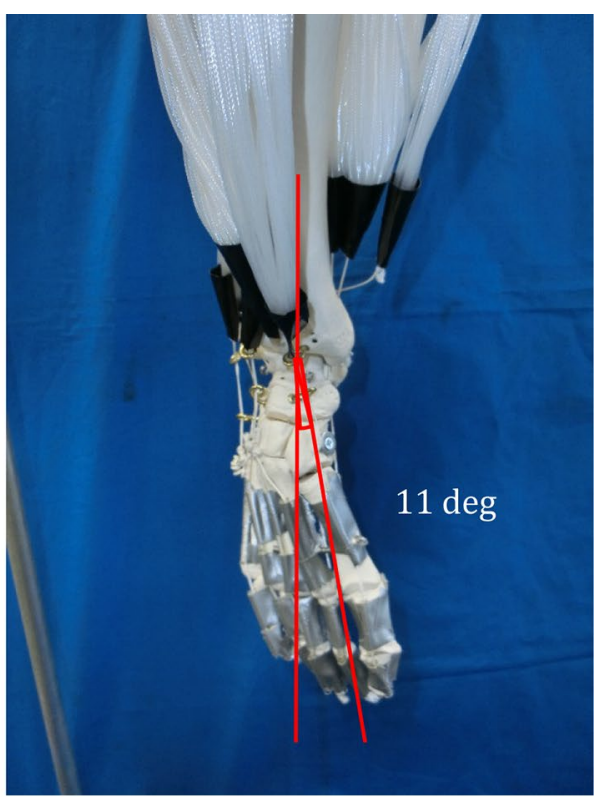

(b)

Fig. 17 a Adduction and $\mathbf{b}$ abduction of an ankle driven by multifilament muscles during inversion or eversion

Table 3 Range of motion of the proposed musculoskeletal robot and human knee joints [16]

\begin{tabular}{lll}
\hline & Musculoskeletal robot & Human \\
\hline Extension (deg) & 120 & 130 \\
Flexion (deg) & 14 & 0 \\
\hline
\end{tabular}

Table 4 Range of motion of the proposed musculoskeletal robot and human knee joints

\begin{tabular}{llll}
\hline & $\begin{array}{l}\text { Musculoskeletal } \\
\text { robot }\left(\mathbf{9 0}^{\circ}\right)\end{array}$ & $\begin{array}{l}\text { Musculoskeletal } \\
\text { robot }\left(\mathbf{1 0 0}^{\circ}\right)\end{array}$ & Human $\left(\mathbf{9 0}^{\circ}\right)$ \\
\hline $\begin{array}{l}\text { Internal rotation } \\
\text { (deg) }\end{array}$ & 15 & - & $10-20$ \\
$\begin{array}{c}\text { External rotation } \\
\text { (deg) }\end{array}$ & - & 26 & $20-30$ \\
\hline
\end{tabular}

The numbers in parentheses indicate the flexion angle during internal or external rotation [16]

systems suitable for the development of a lower-limb musculoskeletal robot. As a result, our robot contains the same number and arrangement of muscles in the human body.
The experiments show that the musculoskeletal driven mechanism achieves motions that are similar to those of a human. The prototype robot demonstrated nearly the same range of motion for both the knee and ankle as a human by using the same muscle arrangement and bone structure. Regarding the knee joint, our robot realized the same degrees of freedom at bending position as a human knee joint by using the same mechanism; two degrees of freedom support both bending and rotation. Our robot also demonstrated the use of an extrinsic muscle to deform a foot like a bow with inversion and eversion (a foot consists of many bones acted upon by an extrinsic muscle). It is the first robot in the world that has realized these human-like characteristics by imitating the human drive mechanism, including the same number and arrangement of muscles in the human body.

While this study evaluated these new motions from a kinematics perspective, we are planning to evaluate this musculoskeletal mechanism in terms of force, stiffness and dynamic characteristics in a subsequent research effort. In addition, this study focused only on knee and ankle motions; future research will consider the application of this method to the entire human body, 


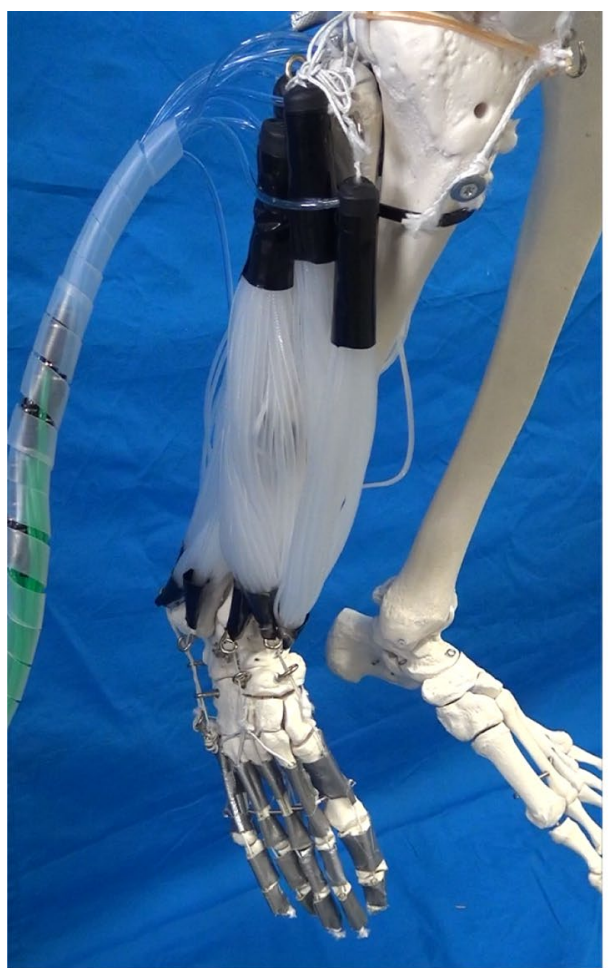

(a)

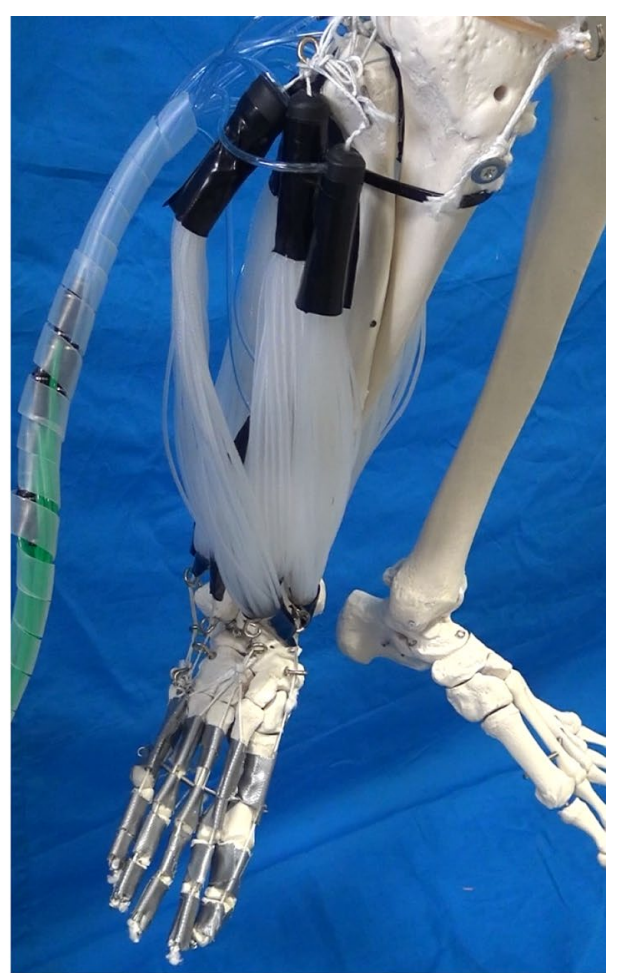

(b)

Fig. 18 a Inversion and $\mathbf{b}$ eversion of an ankle driven by multifilament muscles

Table 5 Range of motion of the proposed musculoskeletal robot and human ankle joints [16]

\begin{tabular}{lll}
\hline & Musculoskeletal robot & Human \\
\hline Dorsal flexion (deg) & 19 & 25 \\
Plantar flexion (deg) & 50 & 50 \\
Pronation (deg) & 30 & 20 \\
Supination (deg) & 40 & 30 \\
Adduction (deg) & 11 & 10 \\
Abduction (deg) & 28 & 20 \\
\hline
\end{tabular}

including the hip and establish a control method for a redundant system to perform practical movements.

\section{Authors' contributions}

All authors equally contributed. All authors read and approved the final manuscript

\section{Author details}

${ }^{1}$ Department of Mechanical and Aerospace Engineering, Tokyo Institute of Technology, Meguro-ku, Ookayama 2-12-1, Tokyo 152-8550, Japan. ${ }^{2}$ Graduate School of Natural Science and Technology, Okayama University, Okayama, Japan.

\section{Acknowledgements}

The present study was supported by JSPS KAKENHI Grant Number 26249028, "Realization of a Next-generation McKibben Artificial Muscles."
Competing interests

The authors declare that they have no competing interests.

Received: 9 February 2016 Accepted: 3 September 2016 Published online: 13 September 2016

\section{References}

1. Asano Y, Mizoguchi H, Osada M, Kozuki T, Urata J, Izawa T, Nakanishi Y, Okada K, Inaba M (2011) Biomimetic design of musculoskeletal humanoid knee joint with patella and screw-home mechanism. In: Proceedings of the 2011 IEEE international conference on robotics and biomimetics, $p$ 1813-1818

2. Asano Y, Mizoguchi H, Kozuki T, Motegi Y, Osada M, Urata J, Nakanishi Y, Okada K, Inaba M (2012) Lower thigh design of detailed musculoskeletal humanoid "Kenshiro." In: Proceedings of the 2012 IEEE/RSJ international conference on intelligent robots and systems, $\mathrm{p} 4367-4372$

3. Marques HG, Jäntsch M, Wittmeier S, Holland O, Alessandro C, Diamond A, Lungarella M, Knight R (2010) ECCE1: The first of a series of anthropomimetic musculoskeletal upper torsos. In: Proceedings of the IEEE-RAS international conference on humanoid robots 2010, p 391-396

4. Holland O, Knight R (2006) The anthropomimetic principle. In: Proceedings of the AISB06 symposium on biologically inspired robotics, $\mathrm{p}$ 1-8

5. Andrikopoulos G, Nikolakopoulos G, Manesis S (2011) A survey on applications of pneumatic artificial muscles. In: Proceedings of the 19th Mediterranean conference on control and automation, p 1439-1446

6. The shadow biped. http://www.shadow.org.uk/projects/biped.shtml. Accessed 7 Aug 2016

7. Narioka K, Niiyama R, Ishii Y, Hosoda K (2009) Pneumatic musculoskeletal infant robots. In: Proceedings of the 2009 IEEE/RSJ International conference on intelligent robots and systems 
8. Takuma T, Hayashi S, Hosoda K (2008) 3D biped robot for multi-modal locomotion driven by antagonistic pneumatic actuators. In: Proceedings of the 4th international symposium on adaptive motion of animals and machines (AMAM2008), Vol 400

9. Boblan I, Schulz A (2010) A humanoid muscle robot torso with biologically inspired construction. In: Proceedings of the 41st International symposium on robotics and ROBOTIK 2010, 6th German conference on robotics

10. Takaoka M, Suzumori K, Wakimoto S, lijima K, Tokumiya T (2013) Fabrication of thin McKibben muscles with various design parameters and their experimental evaluations. In: Proceedings of the 5th international conference on manufacturing, machine design and tribology (ICMDT 2013), p 82

11. Osada M, Mizoguchi H, Asano Y, Kozuki T, Urata J, Nakanishi Y, Okada K, Inaba M (2011) Design of humanoid body trunk with "multiple spine structure" and "planar-muscle-driven" system for achievement of humanlike powerful and lithe motion. In: Proceedings of the 2011 IEEE international conference on robotics and biomimetics, p 2217-2222
12. Kahle VW, Leonhardt H, Platzer W, translator Oti J (1990) Kaibougaku Atlas taschenatlas der anatomie. ver 3, p 17, Bunnkoudou Co., Ltd, Tokyo

13. Doi T, Wakimoto S, Suzumori K, Kanda T (2015) Research on bundle mechanism of thin McKibben artificial muscles -1st report: static characteristics of contraction ratio and contraction force. In: Proceeding of the 2015 JSME conference on robotics and mechatronics, 1P1-B03

14. Netter F (2011) Atlas of human anatomy, 5th edn. Elsevier Japan KK, Tokyo

15. Kawakami K, Isogai K (2013) Anatomy and surface anatomy of muscles, 2nd edn. Daihokaku, Kumamoto

16. Kawashima T, Kuriyama S (2014) Kinniku kansetsu no ugoki to sikumi jiten. Narumido Publishing Company, Tokyo

\section{Submit your manuscript to a SpringerOpen ${ }^{\circ}$ journal and benefit from:}

- Convenient online submission

- Rigorous peer review

- Immediate publication on acceptance

- Open access: articles freely available online

- High visibility within the field

- Retaining the copyright to your article 